Niklas Mattsson, MD, $\mathrm{PhD}$

Henrik Zetterberg, MD, $\mathrm{PhD}$

Shorena Janelidze, $\mathrm{PhD}$

Philip S. Insel, MS

Ulf Andreasson, $\mathrm{PhD}$

Erik Stomrud, MD, PhD

Sebastian Palmqvist, MD,

$\mathrm{PhD}$

David Baker, PhD

Cristina A. Tan Hehir, $\mathrm{PhD}$

Andreas Jeromin, $\mathrm{PhD}$

David Hanlon, $\mathrm{PhD}$

Linan Song, $\mathrm{PhD}$

Leslie M. Shaw, PhD

John Q. Trojanowski, $\mathrm{MD}, \mathrm{PhD}$

Michael W. Weiner, MD

Oskar Hansson, MD,

$\mathrm{PhD}$

Kaj Blennow, MD, PhD

On behalf of the ADNI

Investigators

Correspondence to

Dr. Mattsson:

niklas.mattsson@med.lu.se

Supplemental data at Neurology.org

\section{Plasma tau in Alzheimer disease}

\section{OPEN}

\section{ABSTRACT}

Objective: To test whether plasma tau is altered in Alzheimer disease (AD) and whether it is related to changes in cognition, CSF biomarkers of AD pathology (including $\beta$-amyloid $[A \beta]$ and tau), brain atrophy, and brain metabolism.

Methods: This was a study of plasma tau in prospectively followed patients with AD ( $n=179)$, patients with mild cognitive impairment $(n=195)$, and cognitive healthy controls ( $n=189)$ from the Alzheimer's Disease Neuroimaging Initiative (ADNI) and cross-sectionally studied patients with $A D(n=61)$, mild cognitive impairment $(n=212)$, and subjective cognitive decline $(n=$ $174)$ and controls $(n=274)$ from the Biomarkers for Identifying Neurodegenerative Disorders Early and Reliably (BioFINDER) study at Lund University, Sweden. A total of 1284 participants were studied. Associations were tested between plasma tau and diagnosis, CSF biomarkers, MRI measures, ${ }^{18}$ fluorodeoxyglucose-PET, and cognition.

Results: Higher plasma tau was associated with AD dementia, higher CSF tau, and lower CSF $A \beta_{42}$, but the correlations were weak and differed between ADNI and BioFINDER. Longitudinal analysis in ADNI showed significant associations between plasma tau and worse cognition, more atrophy, and more hypometabolism during follow-up.

Conclusions: Plasma tau partly reflects AD pathology, but the overlap between normal aging and $A D$ is large, especially in patients without dementia. Despite group-level differences, these results do not support plasma tau as an AD biomarker in individual people. Future studies may test longitudinal plasma tau measurements in AD. Neurology ${ }^{\circledR}$ 2016;87:1827-1835

\section{GLOSSARY}

$\mathbf{A} \boldsymbol{\beta}=\beta$-amyloid $; \mathbf{A D}=$ Alzheimer disease; $\mathbf{A D A S}=$ Alzheimer's Disease Assessment Scale-Cognitive subscale; $\mathbf{A D N I}=$ Alzheimer's Disease Neuroimaging Initiative; BioFINDER = Biomarkers for Identifying Neurodegenerative Disorders Early and Reliably; $\mathbf{C I}=$ confidence interval; $\mathbf{C N}=$ cognitively healthy control; DSM-III-R = Diagnostic and Statistical Manual of Mental Disorders, 3rd Edition, Revised; EPIC = European Prospective Investigation Into Cancer and Nutrition; FDG = 18fluorodeoxyglucose; $\mathbf{M C I}=$ mild cognitive impairment; MMSE = Mini-Mental State Examination; P-tau = phosphorylated tau; T-tau = total tau.

Brain accumulation of $\beta$-amyloid $(A \beta)$ and phosphorylated tau (P-tau) in Alzheimer disease (AD) may be monitored by CSF and PET biomarkers. ${ }^{1}$ These technologies have revolutionized clinical research and trials and are rapidly transforming clinical practice. ${ }^{2}$ However, they are hampered by their relative invasiveness and high costs. There is a great unmet need

\footnotetext{
From the Clinical Memory Research Unit (N.M., S.J., P.S.I., E.S., S.P., O.H.), Department of Clinical Sciences, Malmö, Lund University; Department of Neurology (N.M., E.S., O.H., S.P.), Skåne University Hospital, Lund; Clinical Neurochemistry Laboratory (H.Z., U.A., K.B.), Institute of Neuroscience and Physiology, Department of Psychiatry and Neurochemistry, Sahlgrenska Academy at the University of Gothenburg, Mölndal Canpus, Sahlgrenska University Hospital, Mölndal, Sweden; Department of Molecular Neuroscience (H.Z.), UCL Institute of Neurology, Queen Square, London, UK; Janssen R\&D (D.B.), Titusville, NJ; Diagnostics and Life Sciences (C.A.T.H.), GE Global Research, Niskayuna, NY; Quanterix Corporation (A.J., D.H., L.S.), Lexington, MA; Department of Pathology and Laboratory Medicine (L.M.S., J.Q.T.), Perelman School of Medicine, University of Pennsylvania, Philadelphia; Center for Imaging of Neurodegenerative Diseases (M.W.W.), Department of Veterans Affairs Medical Center; and Department of Radiology and Biomedical Imaging (M.W.W.), University of California, San Francisco.

Data used in preparation of this article were obtained from the Alzheimer's Disease Neuroimaging Initiative (ADNI) database (adni.loni.usc.edu). As such, the investigators within the ADNI contributed to the design and implementation of ADNI and/or provided data but did not participate in analysis or writing of this report. A complete listing of ADNI investigators is available at Neurology.org.

Go to Neurology.org for full disclosures. Funding information and disclosures deemed relevant by the authors, if any, are provided at the end of the article. The Article Processing Charge was paid by the authors.

This is an open access article distributed under the terms of the Creative Commons Attribution-NonCommercial-NoDerivatives License 4.0 (CC BY-NC-ND), which permits downloading and sharing the work provided it is properly cited. The work cannot be changed in any way or used commercially.
} 
for less invasive and cheaper biomarkers of $\mathrm{AD}$ pathology. Plasma tau is a candidate because tau is a brain-specific protein that may be expected to leak from brain interstitial fluid to the plasma compartment in AD. Most studies of plasma tau in $\mathrm{AD}$ have been small, including up to $\approx 150$ participants, ${ }^{3-8}$ with one study including 273 participants. ${ }^{9}$ The results have been contradictory, showing clearly elevated levels in $\mathrm{AD}$ (several studies from one group ${ }^{3,6,8}$ ), mild elevations in $\mathrm{AD},{ }^{7}$ no difference between patients with $\mathrm{AD}$ and controls, ${ }^{9}$ or reduced levels in $\mathrm{AD} .{ }^{4,5}$

To clarify the role of plasma tau in $\mathrm{AD}$, we used an ultrasensitive digital ELISA method in 2 large cohorts with 563 participants from a prospective North American study and 721 participants from a cross-sectional Swedish study. We tested the hypotheses that plasma tau is elevated in $\mathrm{AD}$ dementia, correlates with reduced CSF $A \beta_{42}$ and increased CSF total tau (T-tau) and P-tau (biomarkers of AD pathology), and correlates with cognitive impairment, brain atrophy, and reduced brain metabolism.

METHODS ADNI study design. As noted on the Alzheimer's Disease Neuroimaging Initiative (ADNI) database, "Data were obtained from the Alzheimer's Disease Neuroimaging Initiative (ADNI) database (adni.loni.usc.edu). The ADNI was launched in 2003 as a public-private partnership, led by Principal Investigator Michael W. Weiner, MD. For up-to-date information, see www.adni-info.org" (http://adni. loni.usc.edu/wp-content/uploads/how_to_apply/ADNI_DSP_ Policy.pdf). ADNI participants have been recruited from $>50$ sites across the United States and Canada. For the current study, we used data accessed at the ADNI database on November 11, 2015.

ADNI participants. Our ADNI cohort consisted of all cognitively healthy controls $(\mathrm{CNs})$, patients with mild cognitive impairment (MCI), and patients with $\mathrm{AD}$ dementia with available baseline plasma tau samples from ADNI-1. Inclusion and exclusion criteria were described in detail previously. ${ }^{10}$ Briefly, all ADNI-1 participants were between the ages of 55 and 90 years, had completed at least 6 years of education, were fluent in Spanish or English, and had no significant neurologic disease other than AD. CNs had a MiniMental State Examination (MMSE) score $\geq 24$ and Clinical Dementia Rating score of 0. Patients with MCI had an MMSE score $\geq 24$, objective memory loss tested by delayed recall of the Wechsler Memory Scale Logical Memory II ( $>1$ SD below the normal mean), a Clinical Dementia Rating score of 0.5 , preserved activities of daily living, and absence of dementia. Patients with AD dementia fulfilled the National Institute of Neurologic and Communicative Disorders and Stroke and the Alzheimer's Disease and Related Disorders Association criteria for probable $\mathrm{AD}^{11}$ and had an MMSE score of 20 to 26 and Clinical Dementia Rating score of 0.5-1.0.

ADNI measurements. Several of the ADNI procedures have been described previously. ${ }^{12,13} \mathrm{CSF} A \beta_{42}$, T-tau, and P-tau were measured with the multiplex xMAP Luminex platform (Luminex Corp, Austin, TX) with the INNOBIA AlzBio3 kit (Fujirebio, Ghent, Belgium). Participants were classified as A $\beta$ positive or -negative with the use of a previously established cutoff (CSF $A \beta_{42}<192 \mathrm{pg} / \mathrm{mL}$ ). ${ }^{13}$ Using this cutoff, we excluded 9 patients with $\mathrm{AD}$ dementia who were $\mathrm{A} \beta$-negative and therefore likely misdiagnosed. Cognition was assessed by MMSE and Alzheimer's Disease Assessment Scale-Cognitive subscale (ADAS-cog; at screening and 6, 12, 18, 24, 36, and 48 months; n: CNs, 189, 181, 175, 0, 168, 157, and 102; MCI, 195, 188, 182, 167, 154, 130, and 68; AD, 179, 167, 151, 4, 131,12 , and 3). Structural brain images were acquired with 1.5T MRI scanners (at screening and 6, 12, 18, 24, 36 and 48 months; n: CNs, 193, 176, 164, 0, 150, 125, and 86; MCI, 195 , $180,169,151,133,96$, and 54; AD, 177, 156, 136, 1, 101, 2, and 1) with T1-weighted MRI scans using a sagittal volumetric magnetization prepared rapid gradient echo sequence. ${ }^{14}$ FreeSurfer was used for quantification of volumes. ${ }^{14}$ Right and left hippocampi were averaged. ${ }^{18}$ Fluorodeoxyglucose (FDG)PET image data were acquired (at screening and 6, 12, 18, 24, 36 and 48 months; n: CNs, 86, 80, 73, 0, 71, 61, and 51; MCI, $96,90,88,80,72,57$, and 29; $\mathrm{AD}, 91,83,71,1,55,1$, and 1) as described previously. ${ }^{15}$ For FDG-PET, we averaged counts of the lateral and medial frontal, anterior, and posterior cingulate, lateral parietal, and lateral temporal regions. The data were obtained from 2005 to 2014 .

BioFINDER study design, participants, and measurements. From the Swedish 4-center Biomarkers for Identifying Neurodegenerative Disorders Early and Reliably (BioFINDER) study, we included $\mathrm{CNs}$, patients without dementia with mild cognitive symptoms characterized as having subjective cognitive decline or MCI, and patients with $\mathrm{AD}$ dementia. Inclusion/exclusion criteria have been described elsewhere. ${ }^{16,17}$ CNs were originally enrolled from the population-based European Prospective Investigation Into Cancer and Nutrition (EPIC) cohort. ${ }^{18}$ The inclusion criteria were age $\geq 60$ years, MMSE score of 28 to 30 , and fluent in Swedish. Exclusion criteria were the presence of subjective cognitive impairment, significant neurologic or psychiatric disease, dementia, or MCI. Patients with subjective cognitive decline and MCI were recruited consecutively and were assessed by physicians with special competence in dementia disorders. The inclusion criteria were as follows: referred to the memory clinics because of cognitive impairment, not fulfilling the criteria for dementia, MMSE score of 24 to 30, age of 60 to 80 years, and fluent in Swedish. The exclusion criteria were cognitive impairment that without doubt could be explained by another condition (other than prodromal dementia), severe somatic disease, and refusing lumbar puncture or neuropsychological investigation. The classification was based on a neuropsychological battery and the assessment of a senior neuropsychologist. Patients with a clinical syndrome of $\mathrm{AD}$ dementia met the DSM-III-R criteria for dementia ${ }^{19}$ and the National Institute of Neurologic and Communicative Disorders and Stroke and the Alzheimer's Disease and Related Disorders Association criteria for AD. ${ }^{11}$ All participants with plasma tau data were included. CSF sampling was done at baseline, following the Alzheimer's Association Flow Chart. ${ }^{20}$ CSF samples were analyzed by ELISAs 


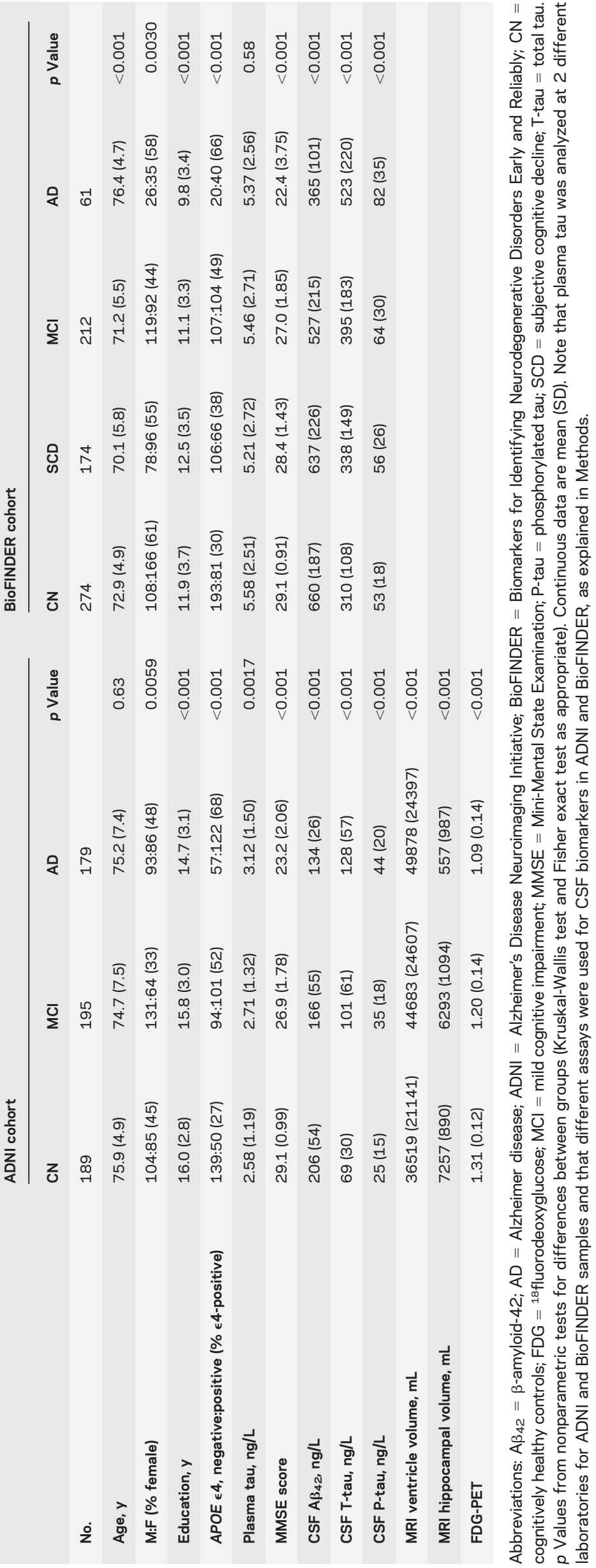

(INNOTEST, Fujirebio, Gent, Belgium, for $A \beta_{42}$ and P-tau; and ADx, EUROIMMUN, Luebeck, Germany, for T-tau). The data were obtained from 2009 to 2015 .

Plasma tau measurements. Plasma tau was analyzed with the Human Total Tau kit (research use only grade, Quanterix, Lexington, MA) on the Simoa HD-1 analyzer (CE marker). This is an updated version of the assay described previously ${ }^{21}$ that uses a monoclonal capture antibody that reacts with a linear epitope in the midregion of all tau isoforms and a detection antibody that reacts with a linear epitope in the $\mathrm{N}$-terminal region of $\mathrm{T}$-tau. The same method (2 different lots) was used for BioFINDER and ADNI. ADNI samples were analyzed at the Clinical Neurochemistry Laboratory, Sahlgrenska University Hospital, Mölndal, Sweden, and BioFINDER samples were analyzed at Quanterix.

Plasma tau was higher in BioFINDER (mean $5.53 \mathrm{ng} / \mathrm{L}$, SD $2.99 \mathrm{ng} / \mathrm{L}$, median $5.11 \mathrm{ng} / \mathrm{L}$, interquartile range $3.78-6.57 \mathrm{ng} /$ L) than in ADNI samples (mean $2.80 \mathrm{ng} / \mathrm{L}, \mathrm{SD} 1.35 \mathrm{ng} / \mathrm{L}$, median $2.65 \mathrm{ng} / \mathrm{L}$, interquartile range $1.88-3.44 \mathrm{ng} / \mathrm{L}$ ). Three ADNI participants ( $1 \mathrm{CN}$ and $2 \mathrm{MCI}$; plasma tau $>10 \mathrm{ng} / \mathrm{L}$ ) and one BioFINDER participant (CN; plasma tau $48.7 \mathrm{ng} / \mathrm{L}$ ) were extreme outliers and were excluded from all analyses. BioFINDER samples had undergone slightly different sample handling than ADNI samples with one less freeze-thaw cycle.

Statistical analysis. First, we tested associations between plasma tau and demographic factors using Mann-Whitney $U$ and Spearman correlation. Second, we tested associations between plasma tau and diagnosis using linear regression adjusted for confounders (with plasma tau as outcome). Third, we tested associations between plasma tau and CSF biomarkers using linear regression adjusted for confounders (with plasma tau as predictor). Fourth, we tested associations between plasma tau and cognition, brain structure, and brain metabolism with longitudinal data using linear mixed-effects models. These models had random intercepts and slopes for time and an unstructured covariance matrix for the random effects and included the interaction between (continuous) time and plasma tau as predictor with adjustment for confounders. To test whether variables were related to study dropout (missing data for cognition, MRI and FDG-PET), we used a generalized mixedeffects model with a binomial response, with plasma tau as predictor of missing data (a missing indicator, true/false, for each study visit). We evaluated interactions between predictors and diagnosis. If they were significant, we performed subgroup analyses within diagnostic groups.

All continuous outcome variables were standardized to facilitate comparisons between modalities. Beta coefficients (which are presented with 95\% confidence intervals [CIs]) from linear regressions and linear mixed effects therefore refer to standardized effects $(\beta=1$ implies that a $1-\mathrm{ng} / \mathrm{L}$ increase in plasma tau was associated with a 1-SD increase in the dependent variable).

We checked model assumptions by inspecting residuals (normality, histograms, q-q plots, linearity, heteroscedasticity, leverage, influence, correlations with fitted values, and predictors of interest). For linear mixed-effects models, we evaluated marginal residuals and conditional residuals. All tests were 2 sided. Significance was determined at $p<0.05$. All statistics were done with $\mathrm{R}$ (version 3.2.3, The R Foundation for Statistical Computing).

Standard protocol approvals, registrations, and patient consents. Regional ethics committees of all institutions approved the ADNI study. The Regional Ethics Committee in 
Figure 1 Plasma tau, diagnosis, and CSF $\beta$-amyloid-42 $\left(A \beta_{42}\right)$

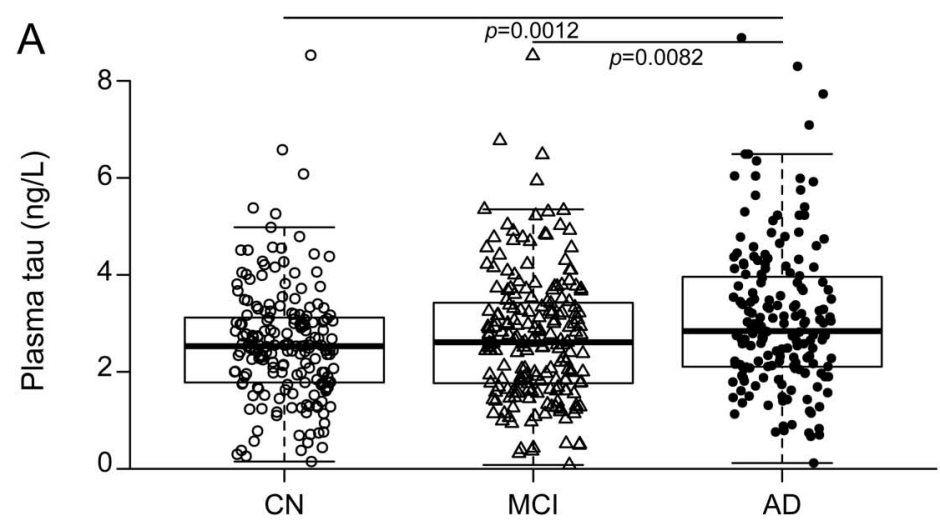

B
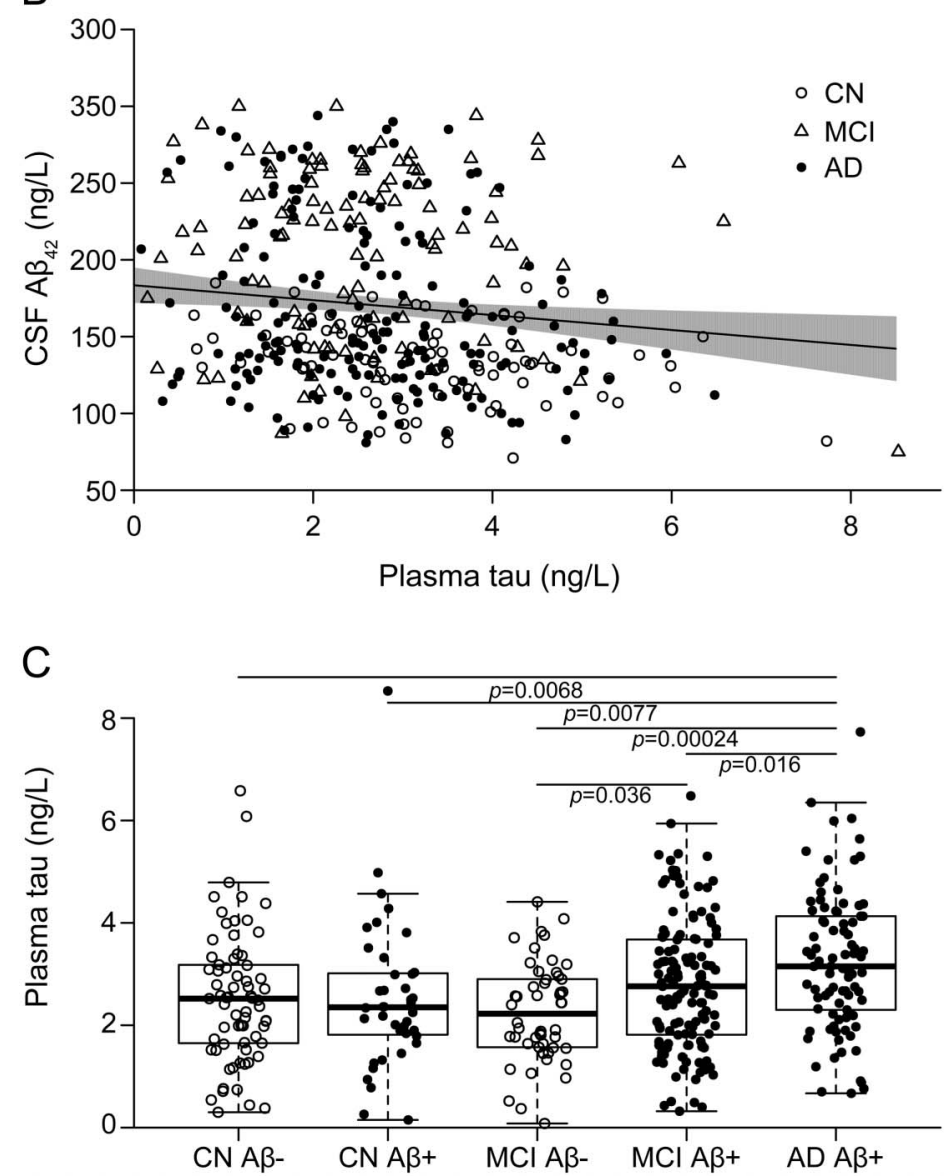

Baseline data from the Alzheimer's Disease Neuroimaging Initiative (ADNI). (A) Plasma tau and diagnosis. $p$ Values from a linear regression model with plasma tau as the dependent variable and diagnosis as a categorical predictor, adjusted for $A P O E \in 4$, age, and sex. Cognitively healthy controls ( $C N s$ ) had lower levels than patients with Alzheimer disease (AD; $\beta=$ $-0.48,95 \%$ confidence interval $[\mathrm{Cl}]-0.19$ to 0.78 ), and patients with mild cognitive impairment (MCl) had lower levels than patients with $A D(\beta=-0.38,95 \% \mathrm{Cl}-0.65$ to 0.098$)$. (B) Plasma tau and CSF $A \beta_{42}$. The trend line is from a linear regression model with plasma tau as the dependent variable and CSF $A_{42}$ as the predictor, adjusted for diagnosis, $A P O E \in 4$, age, and sex. The shaded area indicates the $95 \% \mathrm{Cl}$. In a sensitivity analysis, we removed the 2 bottom-right observations. This affected the slope, but plasma tau and CSF A $\beta_{42}$ were still correlated ( $\beta=-3.99,95 \% \mathrm{Cl}-7.68$ to $0.30, p=0.034$ compared to $\beta=-4.86,95 \% \mathrm{Cl}$ -8.38 to $1.33, p=0.0071$ with all observations included). The $\beta$ coefficients are on the original scale of CSF $A \beta_{42}$; see table 2 for coefficients using standardized data. (C) Plasma tau in diagnostic groups stratified by CSF $A \beta_{42}$ (A $\beta$-positive, CSF $A \beta_{42}<192 \mathrm{ng} / \mathrm{L}$, closed circles; $A \beta$-negative, CSF $A \beta_{42}>192 \mathrm{ng} / \mathrm{L}$, open circles). $p$ Values from a linear regression
Lund, Sweden, approved the BioFINDER study. All study participants gave written informed consent.

RESULTS Table 1 provides demographics. High plasma tau was associated with missing data for $\operatorname{MMSE}(p=0.0017)$, ADAS-cog $(p=0.00047)$, and MRI $(p=0.036)$. This was driven by the patients with AD dementia, who had high plasma tau levels and shorter follow-up than the other groups (see above). The effects of plasma tau on longitudinal cognition and volume may therefore be slightly underestimated, although mixed-effect models are robust to missing data associated with covariates. ${ }^{22}$

Plasma tau and demographic factors. Plasma tau did not correlate with age (ADNI: $\rho=0.042, p=0.32$; BioFINDER: $\rho=-0.012, p=0.74$ ), sex (ADNI: median $2.67 \mathrm{ng} / \mathrm{L}$ for men vs $2.64 \mathrm{ng} / \mathrm{L}$ for women, $p=0.51$; BioFINDER: $4.95 \mathrm{ng} / \mathrm{L}$ for men vs $5.24 \mathrm{ng} / \mathrm{L}$ for women, $p=0.16$ ) or education (ADNI: $\rho=-0.067, p=0.11$; BioFINDER: $\rho=$ $-0.044, p=0.24)$. Plasma tau was slightly increased in $A P O E \in 4$ carriers compared with noncarriers in ADNI (2.75 vs $2.55 \mathrm{ng} / \mathrm{L}, p=0.022)$ but not in BioFINDER (5.10 vs $5.11 \mathrm{ng} / \mathrm{L}, p=0.70$ ).

Plasma tau in different diagnostic groups. In ADNI, plasma tau was higher in patients with $\mathrm{AD}$ dementia (figure 1A) compared with $\mathrm{CNs}$ and patients with MCI. In contrast, plasma tau did not differ between patients with $\mathrm{MCI}$ and $\mathrm{CNs}(\beta=-0.11,95 \% \mathrm{CI}$ -0.38 to $0.17, p=0.44$ ) or between patients with progressive MCI (diagnosed with $\mathrm{AD}$ dementia during the 9-year follow-up, $\mathrm{n}=105)$ and $\mathrm{CNs}(\beta=$ $-0.12,95 \%$ CI -0.42 to $0.17, p=0.41$; results from a model restricted to progressive participants with $\mathrm{MCI}$ and $\mathrm{CNs}$ ). All comparisons were done with linear regressions adjusted for $A P O E \in 4$, age, and sex.

Plasma tau and biomarkers of $\mathbf{A} \boldsymbol{\beta}$ pathology. High plasma tau correlated weakly with low CSF $A \beta_{42}$ in ADNI (figure $1 \mathrm{~B}$, table 2). There was no interaction between plasma tau and diagnosis of CSF $A \beta_{42}$; thus, we did not test effects within diagnostic groups. However, in the whole study cohort, we compared

model testing the effect of the combination of diagnostic group and $A \beta$ status to predict plasma tau, adjusted for $A P O E \in 4$, age, and sex. Patients with AD had higher plasma tau levels than $A \beta$-negative $\mathrm{CNs}(\beta=0.62,95 \% \mathrm{Cl} 1.07-$ 0.17), $A \beta$-positive CNs ( $\beta=0.67,95 \% \mathrm{Cl} 1.16-0.18), A \beta-$ negative patients with $\mathrm{MCl}(\beta=0.90,95 \% \mathrm{Cl} 1.37-0.42)$, and $\mathrm{A} \beta$-positive patients with $\mathrm{MCl}(\beta=0.43,95 \% \mathrm{Cl} 0.78$ 0.081 ), and $A \beta$-positive patients with $\mathrm{MCl}$ had higher plasma tau levels than $A \beta$-negative patients with $\mathrm{MCl}(\beta=$ $0.47,95 \% \mathrm{Cl} 0.90-0.031)$. 


\begin{tabular}{|llc|}
\hline Table 2 & $\begin{array}{l}\text { Associations between plasma tau and Alzheimer disease hallmarks in } \\
\text { the Alzheimer's Disease Neuroimaging Initiative }\end{array}$ & $\boldsymbol{p}$ Value \\
Outcome & $\boldsymbol{\beta}$ Coefficient $(95 \% \mathrm{Cl})$ & 0.0071 \\
CSF A $\beta_{42}$ & $-0.087(-0.15$ to -0.024$)$ & 0.087 \\
CSF T-tau & $0.063(-0.0092$ to 0.14$)$ & 0.20 \\
CSF P-tau & $0.047(-0.025$ to 0.12$)$ & 0.15 \\
MMSE BL & $-0.017(-0.041$ to 0.0063$)$ & $<0.001$ \\
MMSE L & $-0.063(-0.090$ to -0.037$)$ & $<0.001$ \\
ADAS-cog BL & $0.064(0.034$ to 0.094$)$ & $<0.001$ \\
ADAS-cog L & $0.060(0.035$ to 0.085$)$ & 0.027 \\
Ventricular volume BL & $0.059(0.0074$ to 0.11$)$ & $<0.001$ \\
Ventricular volume L & $0.018(0.010$ to 0.025$)$ & 0.87 \\
Hippocampal volume BL & $0.0035(-0.039$ to 0.046$)$ & 0.049 \\
Hippocampal volume L & $-0.0063(-0.013$ to -0.000047$)$ & 0.63 \\
FDG-PET BL & $-0.016(-0.081$ to 0.049$)$ & $<0.001$ \\
FDG-PET L & $-0.039(-0.060$ to -0.019$)$ & \\
\hline
\end{tabular}

Abbreviations: $A \beta_{42}=\beta$-amyloid-42; ADAS-cog $=$ Alzheimer's Disease Assessment ScaleCognitive subscale; $\mathrm{BL}=$ baseline; $\mathrm{Cl}=$ confidence interval; $\mathrm{FDG}=18$ fluorodeoxyglucose; $\mathrm{L}=$ longitudinal; $\mathrm{P}$-tau $=$ phosphorylated tau; $\mathrm{T}$-tau $=$ total tau.

Results are for linear regression (CSF $A_{42}$, CSF T-tau, and CSF P-tau) and linear mixedeffects models (MMSE, ADAS-cog, ventricular volume, hippocampal volume, FDG-PET). Plasma tau was the predictor, adjusted for age, sex, diagnostic group, APOE $\epsilon 4$, and education plus intracranial volume (for ventricular and hippocampal volume). All outcome measures are standardized to facilitate comparison between estimates.

plasma tau between diagnostic groups stratified by $A \beta$, comparing $A \beta$-negative and -positive $C N s, A \beta$ negative and -positive patients with $\mathrm{MCI}$, and $\mathrm{A} \beta$ positive patients with $\mathrm{AD}$ (all participants tested simultaneously by linear regression adjusted for $A P O E \epsilon 4$, age, and sex). Patients with AD dementia had higher plasma tau than all other groups, and $A \beta$ positive patients with MCI had higher plasma tau than $A \beta$-negative patients with MCI (figure $1 C$ ).

Plasma tau and biomarkers of brain tau pathology. Plasma tau did not correlate significantly with CSF T-tau or CSF P-tau in ADNI (table 2). We tested the interactions for plasma tau by diagnostic group. There was a borderline significance for CSF P-tau, which indicated a possible difference between $\mathrm{CNs}$ and patients with $\mathrm{MCI}$ for the association between plasma tau and CSF P-tau $(p=0.056)$. In a subgroup analysis, which should be regarded as exploratory, we found associations in the MCI group between plasma tau and CSF T-tau ( $\beta=0.12,95 \%$ CI 0.0089-0.24, $p=0.035)$ and CSF P-tau $(\beta=0.13,95 \% \mathrm{CI}$ $0.016-0.25, p=0.025)$.

Plasma tau and cognition, atrophy, and brain metabolism. Associations between plasma tau and MMSE score, ADAS-cog, ventricular volume, hippocampal volume, and FDG-PET in ADNI are presented in table 2 and figure 2 . High plasma tau was associated with accelerated decline of MMSE score, high (poor) baseline ADAS-cog and accelerated increase, large baseline ventricular volume and accelerated increase, accelerated decrease of hippocampal volume, and accelerated decline of FDG-PET. Interactions for plasma tau by diagnostic group were significant for MMSE, ADAS-cog, and ventricular volume. We therefore tested models within diagnostic groups for these outcomes. High plasma tau was associated with low baseline MMSE score in $\mathrm{AD}(\beta=-0.062,95 \%$ CI -0.11 to $0.019, p=0.0055)$ and accelerated decline of MMSE score in MCI $(\beta=-0.063$, 95\% CI -0.11 to $0.018, p=0.0068$ ), high baseline ADAS- $\operatorname{cog}(\beta=0.82,95 \%$ CI 0.24-1.40, $p=0.0059)$ and accelerated increase of ADAS-cog in $\mathrm{AD}(\beta=0.81,95 \%$ CI $0.28-1.35, p=0.0031)$, and accelerated increase of ventricular volume in $\mathrm{AD}(\beta=$ $0.026,95 \%$ CI $0.013-0.040, p<0.001$ ). All linear mixed-effects models were adjusted for diagnosis, education, age, sex, and $A P O E \in 4$.

Plasma tau in the BioFINDER cohort. In BioFINDER, plasma tau did not vary by diagnosis $(p=0.16-0.83$ for comparisons between different groups) or CSF $\mathrm{A} \beta_{42}(p=0.89)$ but correlated with CSF T-tau and P-tau $(\beta=0.039,95 \%$ CI 0.013-0.065, $p=$ 0.0033 ; and $\beta=0.032,95 \%$ CI $0.0054-0.059, p=$ 0.019 ; figure 3 ). To determine whether the associations between plasma tau and CSF biomarkers differed by diagnostic group, we tested the interaction between plasma tau and diagnosis, which was present for AD and CSF T-tau $(p=0.0051)$, and there was a tendency for an association between AD and CSF P-tau $(p=0.051)$. This indicated that the relationship between plasma tau and CSF tau biomarkers differed between $\mathrm{CNs}$ and $\mathrm{AD}$, and we found associations only in $\mathrm{AD}$ dementia (CSF T-tau, $\beta=0.13$, 95\% CI 0.039-0.22, $p=0.0057$; CSF P-tau, $\beta=$ $0.093,95 \%$ CI $0.00058-0.19, p=0.049$ ). Plasma tau was not associated with MMSE score $(p=0.77)$. Models were adjusted for age, sex, $A P O E € 4$, diagnosis, and (for MMSE) education.

DISCUSSION We evaluated an ultrasensitive method for tau in plasma and found several associations supporting that plasma tau partly reflects brain pathology. High plasma tau was found in patients with $\mathrm{AD}$ dementia compared to $\mathrm{CNs}$ and patients with MCI (in ADNI), was associated with low CSF $A \beta_{42}$ (in $A D N I$ ), was associated with high CSF tau biomarkers (in BioFINDER), was found in $A P O E \epsilon 4$ carriers (in $\mathrm{ADNI}$ ), and was associated with poor cognition at baseline and accelerated worsening of cognition (in ADNI), large ventricular volumes at baseline and accelerated atrophy (in ADNI), and accelerated decrease in cortical glucose metabolism 
A

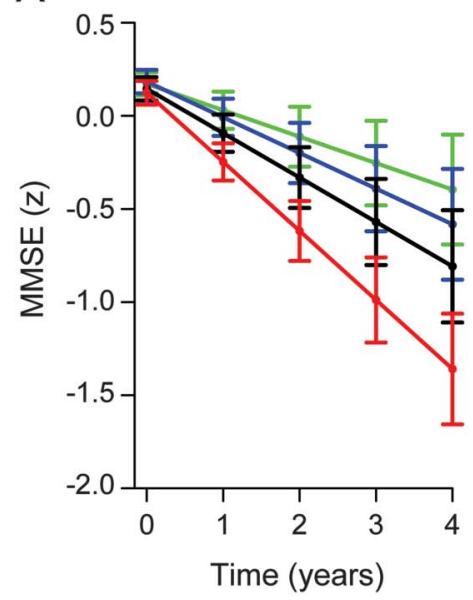

E

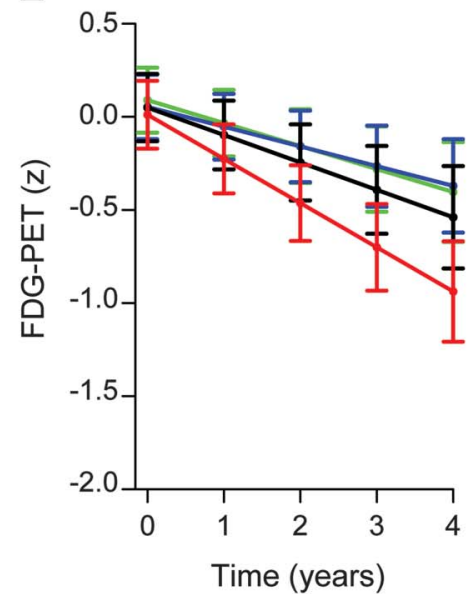

B

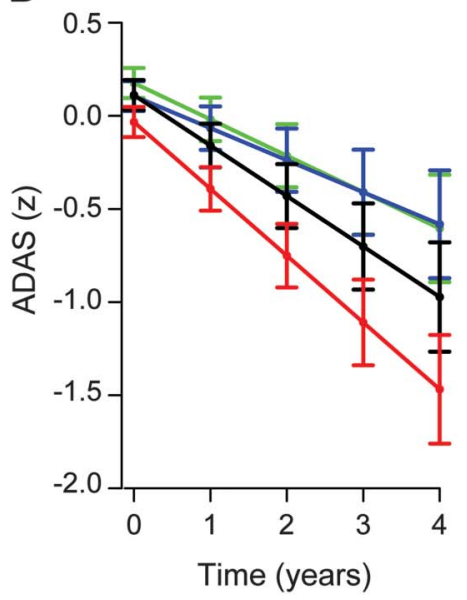

D

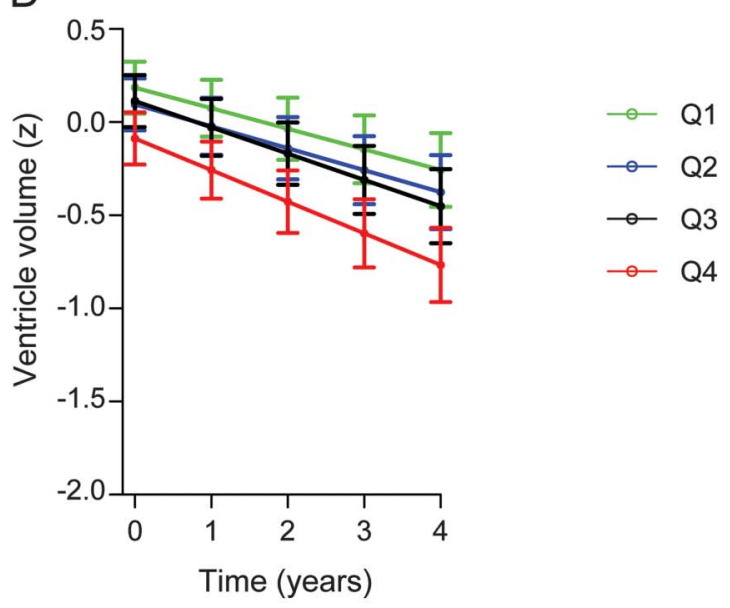

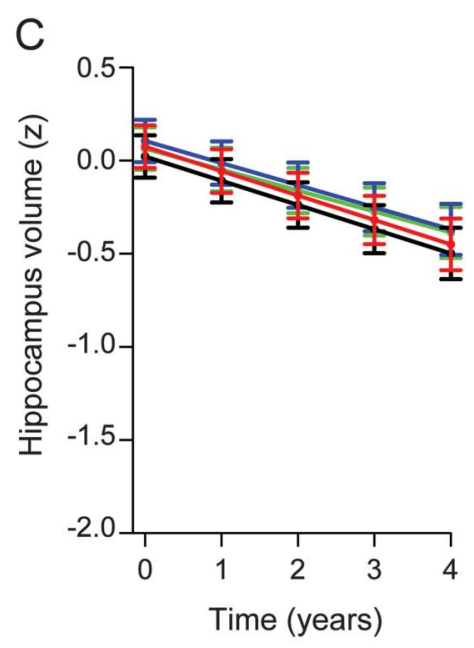
(2)

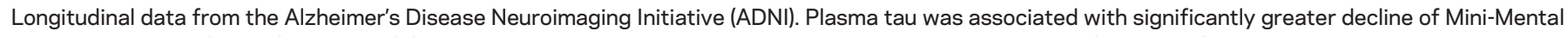

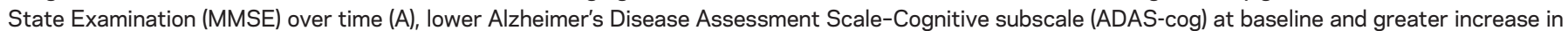

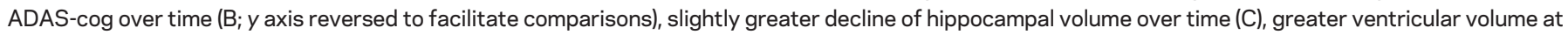

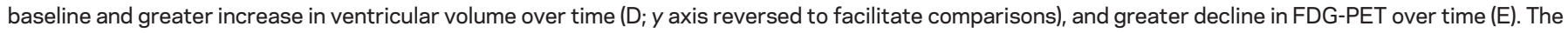

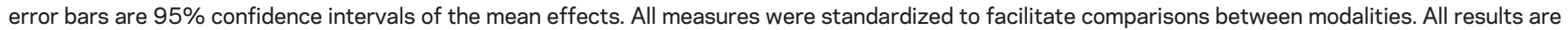

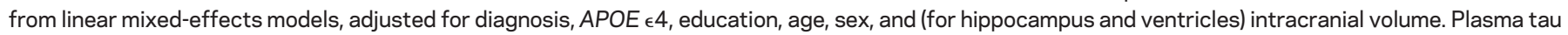

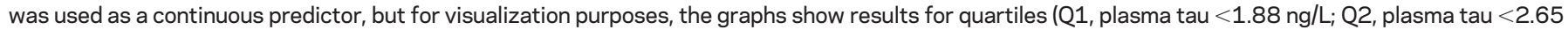
$\mathrm{ng} / \mathrm{L} ; \mathrm{Q} 3$, plasma tau $<3.44 \mathrm{ng} / \mathrm{L} ; \mathrm{Q} 4$, plasma tau $<8.89 \mathrm{ng} / \mathrm{L})$.

(in ADNI). No associations were seen in unexpected or illogical directions. The results suggest that plasma tau partly reflects AD pathology, but the mild associations and the varying results between the cohorts suggest that plasma tau alone is insufficient as an $\mathrm{AD}$ biomarker. This is different from CSF tau, which has high diagnostic accuracy for AD. ${ }^{23}$

Patients with AD had increased plasma tau in ADNI, but this was not replicated in BioFINDER. The small increase in patients with $\mathrm{AD}$ was similar to that in a previous study on another cohort, ${ }^{7}$ while other studies failed to detect differences between CNs, patients with $\mathrm{MCI}$, and patients with $\mathrm{AD}^{9}$ or found reduced plasma tau in patients with $\mathrm{AD}$ compared with CNs, patients with $\mathrm{MCI},{ }^{4}$ or those with vascular dementia. ${ }^{5}$ One group used an immunomagnetic reduction assay for plasma tau ${ }^{8}$ with high separation between $\mathrm{CNs}$ and patients with $\mathrm{AD}$ (sensitivity and specificity $\left.>90 \% 0^{3,6,8}\right)$. Those findings await replication by independent groups.

Plasma tau was associated with CSF biomarkers of $A \beta$ and tau. The associations were weak and differed between ADNI and BioFINDER. In ADNI, high plasma tau was associated with low CSF $A \beta_{42}$, and patients with $\mathrm{AD}$ had higher plasma tau than all other groups, including $A \beta$-positive patients with $\mathrm{MCI}$, who had higher plasma tau than $A \beta$-negative patients with MCI. The association with $A \beta$ was not seen in BioFINDER, in which high plasma tau was instead associated with slightly increased CSF T-tau and P-tau. In ADNI, there was a tendency for an association between plasma tau and CSF T-tau. Together, 
Figure $3 \quad$ Plasma tau and CSF total tau (T-tau) and phosphorylated P-tau

A
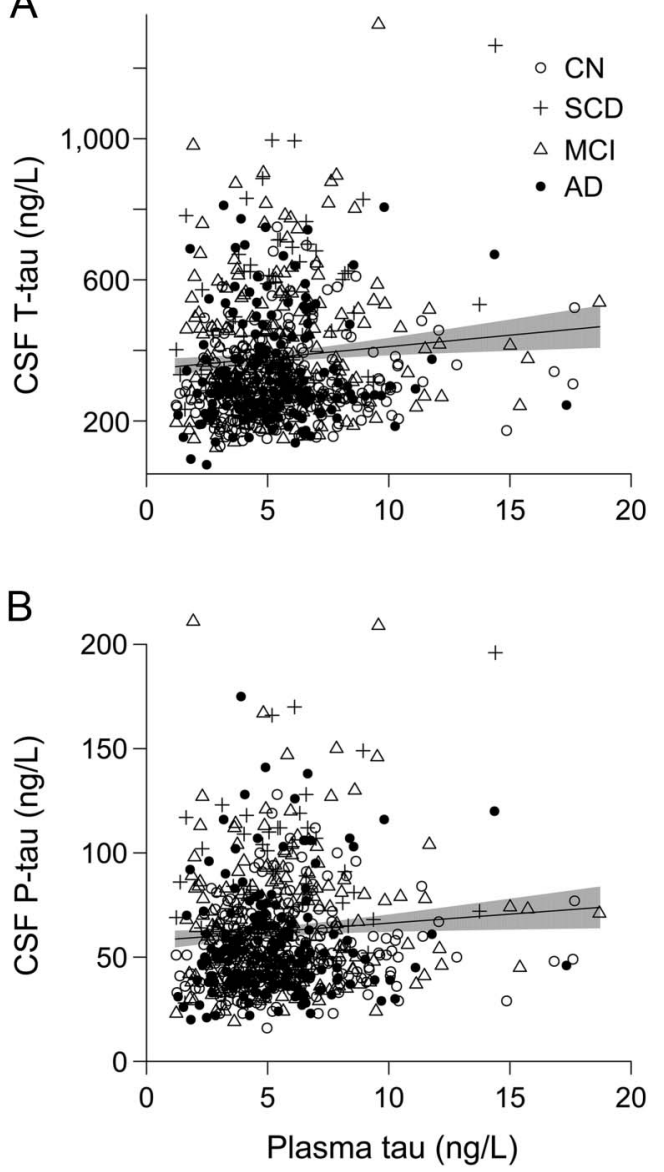

Data from Biomarkers for Identifying Neurodegenerative Disorders Early and Reliably (BioFINDER). The trend lines are for the associations between plasma tau and CSF Ttau $(\beta=6.46,95 \%$ confidence interval $[C l] 2.17-10.76$, $p=0.0033 ; A)$ and $P$-tau $(\beta=0.87,95 \% \mathrm{Cl} 0.15-1.58$, $p=0.034 ; B$ ) in linear regression models adjusted for APOE $\epsilon 4$, diagnosis, age, and sex. The shaded areas indicate $95 \%$ $\mathrm{Cl}$. The $\beta$ coefficients are on the original scales of CSF T-tau and $P$-tau; see the text for coefficients using standardized data. $A D=$ Alzheimer disease; $C N=$ cognitively healthy controls; $\mathrm{MCl}=$ mild cognitive impairment; $\mathrm{SCD}=$ subjective cognitive decline.

these findings suggest that plasma tau captures some information about AD pathology. Previous smaller studies failed to find associations between plasma tau and CSF tau ${ }^{7}$ or PET A $\beta$ biomarkers. ${ }^{6}$

High plasma tau was associated with cognitive impairment, brain atrophy, and brain hypometabolism in ADNI. The associations were significant at baseline (for ADAS-cog and ventricular volume) and over time (for MMSE, ADAS-cog, hippocampal volume, ventricular volume, and FDG-PET) and were seen mainly in $\mathrm{AD}$ and MCI. This suggests that elevated plasma tau is related to disease activity particularly in late clinical stages. Few studies have reported these analyses for plasma tau. One study using the immunomagnetic reduction assay found associations between high plasma tau and small volume of gray matter, hippocampus, and amygdala, but it is not clear if those associations were corrected for diagnostic group. ${ }^{3}$

This study is the largest published on plasma tau, but it is not without limitations. ADNI and BioFINDER samples were handled with different protocols and analyzed at different laboratories with different kit lots, which may have contributed to the varying results. Some ADNI participants had plasma tau below the lower limit of quantification of the assay (14 CNs, 11 patients with MCI, 10 patients with $\mathrm{AD}$ ). These measurements are uncertain but they were included because excluding them would have biased the data toward higher plasma tau (excluding them did not change the main results, data not shown). Another limitation is that we used only one plasma tau assay. It is possible that other assays capture tau fragments that are less sensitive to peripheral degradation and more likely to reflect $\mathrm{AD}$ pathology. A final limitation is that we did not covary for copathologies that may affect plasma tau levels.

We found associations between elevated plasma tau and $\mathrm{AD}$ hallmarks, but the associations were mild and differed between cohorts. Our longitudinal data suggest that high plasma tau is associated with rapid progression in later disease stages. The large study sample supports generalization of these results. Future studies should include longitudinal measurements to explore whether plasma tau is sensitive to intraindividual changes during the progression of $\mathrm{AD}$ and an assessment of plasma tau across neurodegenerative diseases. This would increase the usability of plasma tau as an AD biomarker, particularly in clinical trials for patient enrichment and stratification.

\section{AUTHOR CONTRIBUTIONS}

Drafting the manuscript: N.M. Revising the manuscript for content: all authors. Study concept or design: N.M., O.H., D.B., C.A.T.H., K.B., H.Z. Analysis or interpretation of data: N.M., O.H., K.B., H.Z. Contribution of vital reagents/tools/patents: D.H., A.J., C.A.T.H., D.B., L.S. Acquisition of data: U.A., S.P., D.H., A.J., E.S., J.Q.T., L.M.S., L.S. Statistical analysis: N.M. Study supervision or coordination: N.M., O.H., K. B., U.A. Obtaining funding: N.M., O.H., D.B., C.A.T.H., K.B., H.Z., M.W.W.

\section{ACKNOWLEDGMENT}

The authors would like to thank the Swedish BioFINDER study group.

\section{STUDY FUNDING}

Data collection and sharing for this project were funded by the ADNI (National Institutes of Health grant U01 AG024904) and DOD ADNI (Department of Defense award number W81XWH-12-20012). ADNI is funded by the National Institute on Aging, the National Institute of Biomedical Imaging and Bioengineering, and through generous contributions from the following: Alzheimer's Association; Alzheimer's Drug Discovery Foundation; Araclon 
Biotech; BioClinica, Inc; Biogen Idec Inc; Bristol-Myers Squibb Company; Eisai Inc; Elan Pharmaceuticals, Inc; Eli Lilly and Company; EuroImmun; F. Hoffmann-La Roche Ltd and its affiliated company Genentech, Inc; Fujirebio; GE Healthcare; IXICO Ltd; Janssen Alzheimer Immunotherapy Research \& Development, LLC; Johnson \& Johnson Pharmaceutical Research \& Development LLC; Medpace, Inc; Merck \& Co, Inc; Meso Scale Diagnostics, LLC; NeuroRx Research; Neurotrack Technologies; Novartis Pharmaceuticals Corporation; Pfizer Inc; Piramal Imaging; Servier; Synarc Inc; and Takeda Pharmaceutical Company. The Canadian Institutes of Health Research is providing funds to support ADNI clinical sites in Canada. Private sector contributions are facilitated by the Foundation for the National Institutes of Health (www.fnih org). The grantee organization is the Northern California Institute for Research and Education, and the study is coordinated by the Alzheimer's Disease Cooperative Study at the University of California, San Diego. ADNI data are disseminated by the Laboratory for Neuro Imaging at the University of Southern California. This research was also supported by grants from the Swedish Alzheimer Foundation, the Greta and Johan Kock Foundation, the Thelma Zoega Foundation, the Torsten Söderberg Foundation at the Roya Swedish Academy of Sciences, the Strategic Research Area of Neuroscience at Lund University (MultiPark), the European Research Council, the Swedish Research Council, the Strategic Research Area MultiPark (Multidisciplinary Research in Parkinson's Disease) at Lund University, the Crafoord Foundation, the Swedish Brain Foundation, the Knut and Alice Wallenberg Foundation, and the Swedish federal government under the ALF agreement. N.M. had full access to all the data in the study and takes responsibility for the integrity of the data and the accuracy of the data analysis. Janssen provided funding for the Quanterix analysis of the BioFINDER cohort. No sponsor had any role in the design and conduct of the study; collection, management, analysis, and interpretation of the data; and preparation, review, or approval of the manuscript; and decision to submit the manuscript for publication.

\section{DISCLOSURE}

N. Mattsson reports no disclosures relevant to the manuscript. H. Zetterberg is a cofounder of Brain Biomarker Solutions in Gothenburg AB, a GU Holding-based platform company at the University of Gothenburg. S. Janelidze, P. Insel, U. Andreasson, E. Stromrud, and S. Palmqvist report no disclosures relevant to the manuscript. D. Baker works for Janssen R\&D, which provided funding for this work. C. Tan Hehir is an employee of General Electric. A. Jeromin is a paid advisor to Quanterix Corp and holds stock options. D. Henlon and L. Song report no disclosures relevant to the manuscript. L. Shaw receives funding from NIH/NIA ADNI U01 grant AG024904 and MJFox Foundation for Parkinson's Disease Research; provides quality control advice to Fujirebio-Europe as part of the ADNI Biomarker Core responsibilities; and is a consultant for Eli Lilly, Novartis, and Janssen. J. Trojanowski may accrue revenue in the future on patents submitted by the University of Pennsylvania wherein he is coinventor and he received revenue from the sale of Avid to Eli Lily as coinventor on imaging-related patents submitted by the University of Pennsylvania. He receives research support from the NIH, GSK, Janssen, Biogen, and several nonprofits. M. Weiner has been on scientific advisory boards for Pfizer and BOLT International; has been a consultant for Pfizer Inc, Janssen, KLJ Associates, Easton Associates, Harvard University, inThought, INC Research, Inc, University of California, Los Angeles, Alzheimer's Drug Discovery Foundation, and Sanofi-Aventis Group; has received funding for travel from Pfizer, AD PD meeting, Paul Sabatier University, Novartis, Tohoku University, MCI Group, France, Travel eDreams, Inc, Neuroscience School of Advanced Studies (NSAS), Danone Trading, BV, and CTAD ANT Congress; serves as an associate editor of Alzheimer's \& Dementia; has received honoraria from Pfizer, Tohoku University, and Danone Trading, BV; has research support from Merck, Avid, Department of Defense, and Veterans Affairs; and has stock options in Synarc and Elan. O. Hansson reports no disclosures relevant to the manuscript. K. Blennow has served as advisory boards for Roche
Diagnostics and IBL International and is a cofounder of Brain Biomarker Solutions in Gothenburg AB, a GU Holding-based platform company at the University of Gothenburg. Go to Neurology.org for full disclosures.

Received October 16, 2015. Accepted in final form July 12, 2016.

\section{REFERENCES}

1. Blennow $K$, Mattsson $N$, Schöll $M$, Hansson $O$, Zetterberg H. Amyloid biomarkers in Alzheimer's disease. Trends Pharmacol Sci 2015;36:297-309.

2. Mattsson N, Carrillo MC, Dean RA, et al. Revolutionizing Alzheimer's disease and clinical trials through biomarkers. Alzheimers Dement Diagn Assess Dis Monit 2015;1:412-419.

3. Chiu MJ, Chen YF, Chen TF, et al. Plasma tau as a window to the brain-negative associations with brain volume and memory function in mild cognitive impairment and early Alzheimer's disease. Hum Brain Mapp 2014;35: 3132-3142.

4. Sparks DL, Kryscio RJ, Sabbagh MN, et al. Tau is reduced in AD plasma and validation of employed ELISA methods. Am J Neurodegener Dis 2012;1:99-106.

5. Krishnan S, Rani P. Evaluation of selenium, redox status and their association with plasma amyloid/tau in Alzheimer's disease. Biol Trace Elem Res 2014;158:158-165.

6. Tzen KY, Yang SY, Chen TF, et al. Plasma A $\beta$ but not tau is related to brain $\mathrm{PiB}$ retention in early Alzheimer's disease. ACS Chem Neurosci 2014;5:830-836.

7. Zetterberg H, Wilson D, Andreasson U, et al. Plasma tau levels in Alzheimer's disease. Alzheimers Res Ther 2013;5:9.

8. Chiu MJ, Yang SY, Horng HE, et al. Combined plasma biomarkers for diagnosing mild cognition impairment and Alzheimer's disease. ACS Chem Neurosci 2013;4:1530-1536.

9. Wang T, Xiao S, Liu Y, et al. The efficacy of plasma biomarkers in early diagnosis of Alzheimer's disease. Int J Geriatr Psychiatry 2014;29:713-719.

10. Petersen RC, Aisen PS, Beckett LA, et al. Alzheimer's disease neuroimaging initiative (ADNI): clinical characterization. Neurology; 2010;74:201-209.

11. McKhann G, Drachman D, Folstein M, Katzman R, Price D, Stadlan EM. Clinical diagnosis of Alzheimer's disease: report of the NINCDS-ADRDA Work Group under the auspices of Department of Health and Human Services Task Force on Alzheimer's Disease. Neurology 1984;34:939-944.

12. Portelius E, Zetterberg H, Skillbäck T, et al. Cerebrospinal fluid neurogranin: relation to cognition and neurodegeneration in Alzheimer's disease. Brain J Neurol 2015;138: 3373-3385.

13. Shaw LM, Vanderstichele H, Knapik-Czajka M, et al. Cerebrospinal fluid biomarker signature in Alzheimer's Disease Neuroimaging Initiative subjects. Ann Neurol 2009;65:403-413.

14. Jack CR Jr, Bernstein MA, Fox NC, et al. The Alzheimer's Disease Neuroimaging Initiative (ADNI): MRI methods. J Magn Reson Imaging JMRI 2008;27:685-691.

15. Landau SM, Mintun MA, Joshi AD, et al. Amyloid deposition, hypometabolism, and longitudinal cognitive decline. Ann Neurol 2012;72:578-586.

16. Palmqvist $S$, Zetterberg $\mathrm{H}$, Blennow K, et al. Accuracy of brain amyloid detection in clinical practice using cerebrospinal fluid $\beta$-amyloid 42: a cross-validation study against 
amyloid positron emission tomography. JAMA Neurol 2014;71:1282-1289.

17. Palmqvist S, Zetterberg H, Mattsson N, et al. Detailed comparison of amyloid PET and CSF biomarkers for identifying early Alzheimer disease. Neurology 2015; 85:1240-1249.

18. Haftenberger M, Schuit AJ, Tormo MJ, et al. Physical activity of subjects aged 50-64 years involved in the European Prospective Investigation into Cancer and nutrition (EPIC). Public Health Nutr 2002;5:1163-1176.

19. American Psychiatric Association. Diagnostic and Statistical Manual of Mental Disorders: DSM-III-R. Washington, DC: American Psychiatric Association; 1987.
20. Blennow K, Hampel H, Weiner M, Zetterberg H. Cerebrospinal fluid and plasma biomarkers in Alzheimer disease. Nat Rev Neurol 2010;6:131-144.

21. Randall J, Mörtberg E, Provuncher GK, et al. Tau proteins in serum predict neurological outcome after hypoxic brain injury from cardiac arrest: results of a pilot study. Resuscitation 2013;84:351-356.

22. Little R, Rubin D. Statistical Analysis With Missing Data. 2nd ed. Hoboken: Wiley; 2002.

23. Bloudek LM, Spackman DE, Blankenburg M, Sullivan SD. Review and meta-analysis of biomarkers and diagnostic imaging in Alzheimer's disease. J Alzheimers Dis 2011;26:627-645.

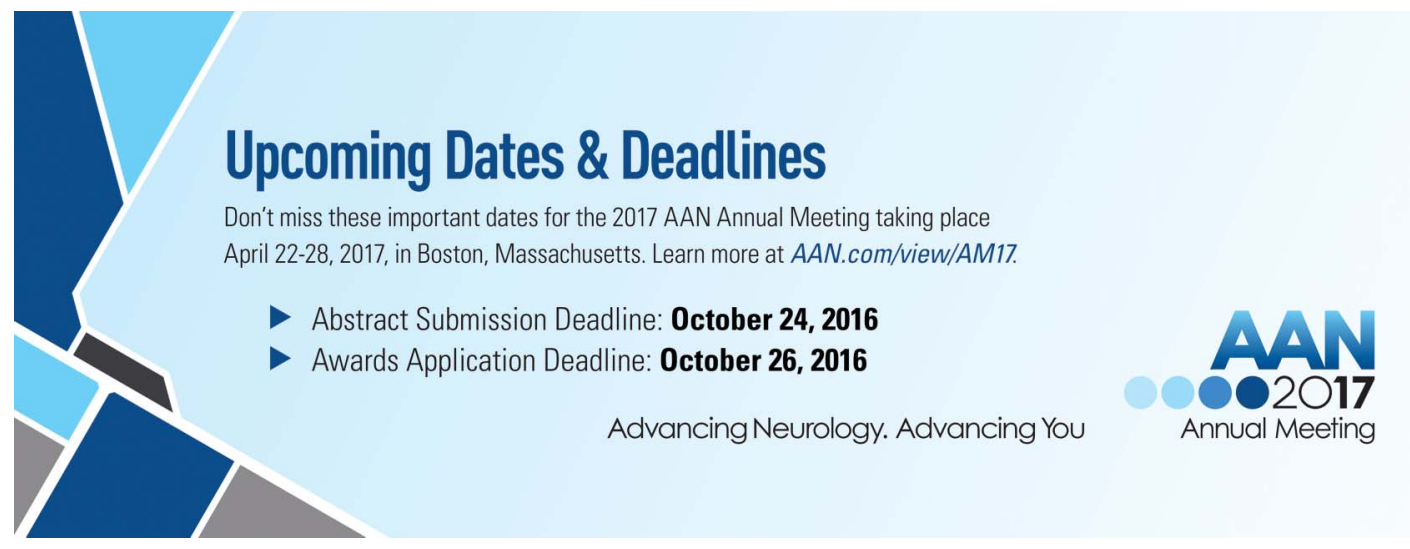

\section{Visit the Neurology ${ }^{\circledR}$ Website at Neurology.org}

- Enhanced navigation format

- Increased search capability

- Highlighted articles

- Detailed podcast descriptions

- RSS Feeds of current issue and podcasts

- Personal folders for articles and searches

- Mobile device download link

- AAN Web page links

- Links to Neurology Now ${ }^{\circledR}$, Neurology Today ${ }^{\circledR}$, and Continuum ${ }^{\circledR}$

- Resident \& Fellow subsite

(1) Fin: Find Neurology ${ }^{\circledR}$ on Facebook: http://tinyurl.com/neurologyfan

twitter Follow Neurology ${ }^{\circledR}$ on Twitter: https://twitter.com/GreenJournal 


\section{Neurology}

\section{Plasma tau in Alzheimer disease}

Niklas Mattsson, Henrik Zetterberg, Shorena Janelidze, et al.

Neurology 2016;87;1827-1835 Published Online before print September 30, 2016

DOI 10.1212/WNL.0000000000003246

This information is current as of September 30, 2016

\section{Updated Information \& Services}

Supplementary Material

\section{References}

Citations

Subspecialty Collections

Permissions \& Licensing

Reprints including high resolution figures, can be found at: http://n.neurology.org/content/87/17/1827.full

Supplementary material can be found at: http://n.neurology.org/content/suppl/2016/09/30/WNL.0000000000003 246.DC1

This article cites 21 articles, 3 of which you can access for free at: http://n.neurology.org/content/87/17/1827.full\#ref-list-1

This article has been cited by 7 HighWire-hosted articles: http://n.neurology.org/content/87/17/1827.full\#\#otherarticles

This article, along with others on similar topics, appears in the following collection(s):

Alzheimer's disease

http://n.neurology.org/cgi/collection/alzheimers_disease

Cerebrospinal Fluid

http://n.neurology.org/cgi/collection/cerebrospinal_fluid MCI (mild cognitive impairment)

http://n.neurology.org/cgi/collection/mci_mild_cognitive_impairment

Information about reproducing this article in parts (figures,tables) or in its entirety can be found online at:

http://www.neurology.org/about/about_the_journal\#permissions

Information about ordering reprints can be found online:

http://n.neurology.org/subscribers/advertise

Neurology ${ }^{\circledR}$ is the official journal of the American Academy of Neurology. Published continuously since 1951, it is now a weekly with 48 issues per year. Copyright @ 2016 American Academy of Neurology. All rights reserved. Print ISSN: 0028-3878. Online ISSN: 1526-632X.

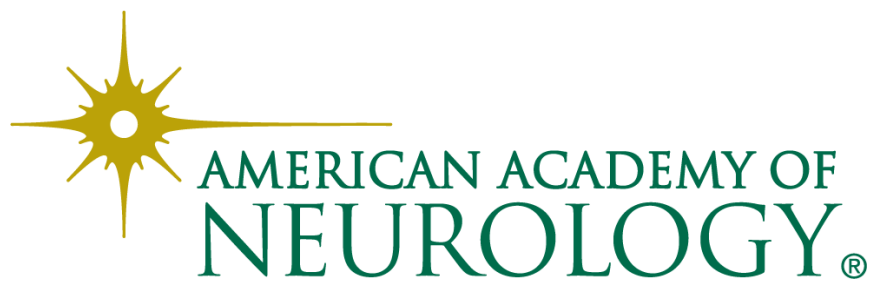

